IML 99-34

hep-th/9911239

\title{
The formulae of Kontsevich and Verlinde from the perspective of the Drinfeld double
}

\author{
C. Klimčík \\ Institute de mathématiques de Luminy, \\ 163, Avenue de Luminy, 13288 Marseille, France
}

\begin{abstract}
A two dimensional gauge theory is canonically associated to every Drinfeld double. For particular doubles, the theory turns out to be e.g. the ordinary Yang-Mills theory, the G/G gauged WZNW model or the Poisson $\sigma$-model that underlies the Kontsevich quantization formula. We calculate the arbitrary genus partition function of the latter. The result is the $q$-deformation of the ordinary Yang-Mills partition function in the sense that the series over the weights is replaced by the same series over the $q$-weights. For $q$ equal to a root of unity the series acquires the affine Weyl symmetry and its truncation to the alcove coincides with the Verlinde formula.
\end{abstract}




\section{Introduction}

The original motivation of this article was to elucidate a relation between the Yang-Mills theory in two dimensions and the $G / G$ gauged WZNW model. It is known [1, 2] that the latter can be understood as a sort of a nonlinear deformation of the former. The first main result of this work shows that it exists a whole moduli space of two dimensional gauge theories which contains the both theories mentioned above as special points.

Throughout this paper, $G$ will denote a simple compact connected and simply connected Lie group. We shall argue that the moduli space of YangMills-like theories in two dimensions based on the group $G$ coincides with the space of doubles $D(G)$ of $G$ and we shall refer to the points in this moduli space as to Poisson-Lie Yang-Mills theories. We define a double $D(G)$ of an $n$-dimensional real Lie group $G$ to be any $2 n$-dimensional real Lie group $D$ (containing $G$ as its subgroup) such that its Lie algebra $\mathcal{D}$ is equipped with an symmetric invariant non-degenerate $\mathbf{R}$-bilinear form $\langle.,$.$\rangle with respect to$ which the Lie algebra $\mathcal{G}$ of $G$ is isotropic (i.e. $\langle\mathcal{G}, \mathcal{G}\rangle=0$ ).

Our second main result is an observation that the Poisson $\sigma$-models, corresponding to the Poisson-Lie structures on group manifolds, are special points in our moduli space. More precisely, they are the Poisson-Lie Yang-Mills theories with vanishing coupling constant. A particular example of the Poisson $\sigma$-model is the BF theory which indeed can be obtained from the ordinary Yang-Mills theory by setting to zero the coupling constant of the latter.

The Poisson $\sigma$-models were introduced by Ikeda [3] and Schaller and Strobl [4] for manifolds without boundary. Their actions read

$$
S=\int_{\Sigma}\left(C_{i} \wedge d X^{i}+\frac{1}{2} \alpha^{i j}(X) C_{i} \wedge C_{j}\right)
$$

Here $X^{i}$ is the set of coordinates on the manifold $M$ viewed as functions on the world-sheet $\Sigma, \alpha^{i j}(X)$ denotes a bivector field (i.e. a section of $\wedge^{2} T M$ ) which defines the Poisson structure on $M$ and $C_{i}$ is a set of 1-form fields on $\Sigma$ which can be interpreted as sections of the bundle $X^{*}\left(T^{*} M\right) \otimes T^{*} \Sigma$.

Recently, Cattaneo and Felder [5] have shown that certain correlators of the Poisson $\sigma$-models, corresponding to insertions at the boundary of the disc, are computed by the Kontsevich formula [6]. This is in the sense of the perturbation expansion in the field theoretic Planck constant $\hbar$. In our picture, this Planck constant $\hbar$ turns out to be the parameter which multiplies 
the Poisson-Lie bracket on $G$ and therefore it can be interpreted as the Planck constant also from this point of view.

The third main result of this paper is the computation of an arbitrary genus partition function of the Poisson-Lie Yang-Mills theory corresponding to the Lu-Weinstein-Soibelman (LWS) Drinfeld double $D(G)$. This model possesses the gauge symmetry based on the group $G$. For the special case of the vanishing coupling constant, we thus obtain the partition function of the Poisson $\sigma$-model for the LWS Poisson-Lie structure on the dual group $\tilde{G}$. If we set $q=e^{2 \pi \hbar B(\psi, \psi)}$, where $B(.,$.$) is the Killing-Cartan form on \mathcal{G}$ and $\psi$ is the longest root, this partition function $Z(q)$ has an interesting behaviour in the complex plane $q$. In fact, for $q=1$ it gives the ordinary $\mathrm{BF}$ partition function and for $q \neq 1$ but equal to a root of unity it gives the standard Verlinde formula [7]. It therefore appears natural to refer to the partition function of the Poisson $\sigma$-model for an arbitrary double $D(G)$ as to a generalized $D(G)$ Verlinde formula.

All those results suggest that the Kontsevich and the Verlinde formulae are in fact cousins; a general correlator of the Poisson $\sigma$-model with bulk and boundary insertions, on arbitraty genus and for arbitrary double $D(G)$ is the object that appears to generalize both of them.

In section 2, we shall define the Poisson-Lie Yang-Mills theory as the gauge theory canonically associated to every double $D(G)$ and we shall indicate the doubles which give respectively the ordinary Yang-Mills theory and the $G / G$ gauged WZNW model. In section 3, we review the definition of the LWS double, we identify its Poisson-Lie Yang-Mills theory and show how the Poisson $\sigma$-model (1) emerges if the coupling constant vanishes. We calculate the corresponding partition function in section 4 . We use an appropriate generalization of the method of Blau and Thompson worked out for the ordinary Yang-Mills theory and for the $G / G$ gauged WZNW model in [2, 8]. We shall finish with a short outlook.

\section{The Poisson-Lie Yang-Mills theory}

The Poisson-Lie Yang-Mills theory, that we shall associate to every double of a Lie group $G$, is simply obtained by an isotropic gauging of the WZNW model on the double. Its action reads

$$
S\left(l, \eta^{10}, \eta^{01}\right)=
$$




$$
I(l)+\frac{1}{2 \pi} \int_{\Sigma}\left[\left\langle\partial_{+} l l^{-1}, \eta^{10}\right\rangle-\left\langle\eta^{01}, l^{-1} \partial_{-} l\right\rangle-\left\langle l \eta^{01}, l^{-1}, \eta^{10}\right\rangle\right]+\varepsilon_{i} \int_{\Sigma} \omega O_{i}(l),
$$

where $l$ is a map from the world-sheet into the double $D$, and $\eta^{10}$ and $\eta^{01}$ are respectively $(1,0)$ and $(0,1)$ forms on the world-sheet with values in the isotropic subalgebra $\mathcal{G}$ of the Lie algebra $\mathcal{D}$ of $D . \varepsilon_{i}$ are coupling constants, $\omega$ is a volume form on the world-sheet and $O_{i}(l)$ are functions on the group manifold $D$ which are separately invariant with respect to the left and right action of $G$ on $D$. $\partial_{-}$and $\partial_{+}$denote the Minkowski version of the Dolbeault coboundary operators, in particular, acting on functions they are given by the standard light-cone derivatives

$$
\partial_{ \pm}=\partial_{\tau} \pm \partial_{\sigma}
$$

Note that the (2) is written entirely in the language of the differential forms, though we have suppressed the symbol of the wedge product. The pure WZNW model action is given in the standard way

$$
I(l)=\frac{1}{4 \pi} \int\left\langle\partial_{+} l l^{-1}, \partial_{-} l l^{-1}\right\rangle+\frac{1}{24 \pi} \int d^{-1}\left\langle d l l^{-1},\left[d l l^{-1}, d l l^{-1}\right]\right\rangle .
$$

Of course, it is obvious how to make sense of (4) also when $D$ is not a matrix group.

In this section and in the following one the Poisson-Lie Yang-Mills theory (2) will be considered only at the classical level; the world-sheet $\Sigma$ will be always a cylinder equipped with the Minkowski metric. This means, that the variation problem with the fixed boundary conditions at the initial and final time is well-defined (see [9] for more details about the WZNW model on the cylinder). When we fix a gauge, we shall always tacitly assume that those initial and final boundary conditions are compatible with this fixing.

The model (2) is gauge invariant with respect to two mutually commuting gauge symmetries:

$$
l \rightarrow g l k^{-1}, \quad \eta^{10} \rightarrow g \eta^{10} g^{-1}-\partial_{-} g g^{-1}, \quad \eta^{01} \rightarrow k \eta^{01} k^{-1}-\partial_{-} k k^{-1},
$$

where $g, k \in G$ are mappings from the world-sheet into the maximally isotropic subgroup $G$. The crucial property of the action (4), which is needed for verifying the gauge invariance (5), is the validity of the PolyakovWiegmann formula [10]:

$$
I\left(l_{1} l_{2}\right)=I\left(l_{1}\right)+I\left(l_{2}\right)+\frac{1}{2 \pi} \int\left\langle\partial_{+} l_{1} l_{1}^{-1}, l_{2}^{-1} \partial_{-} l_{2}\right\rangle
$$


Let us consider a compact group $G$ and for its double $D(G)$ we take its cotangent bundle $T^{*} G$. This bundle is of course trivializable, hence we can represent every point in its total space as a pair $(g, X)$, where $g \in G$ and $X \in \mathcal{G}^{*}$. The group law is then

$$
\left(g_{1}, X_{1}\right)\left(g_{2}, X_{2}\right)=\left(g_{1} g_{2}, \operatorname{Coad}_{g_{1}} X_{2}+X_{1}\right)
$$

and the Lie algebra of $T^{*} G$ is the semidirect sum of $\mathcal{G}$ and $\mathcal{G}^{*}$, where $\mathcal{G}$ acts on $\mathcal{G}^{*}$ in the coadjoint way. Finally, the invariant nondegenerate bilinear form $\langle.,$.$\rangle on \mathcal{D}$ is given by

$$
\langle(\alpha, X),(\beta, Y)\rangle \equiv Y(\alpha)+X(\beta), \quad \alpha, \beta \in \mathcal{G}, \quad X, Y \in \mathcal{G}^{*} .
$$

If we now partially fix the gauge by setting $l=(1, X)$, we obtain from (2) the following theory

$$
S=-\frac{1}{2 \pi} \int_{\Sigma} X(d \eta+\eta \wedge \eta)+\varepsilon_{i} \int_{\Sigma} \omega O_{i}(X)
$$

where $\eta=\eta^{10}+\eta^{01}$. If we set all $\varepsilon_{i}$, but $\varepsilon_{1}$, to zero and choose

$$
O_{1}(X)=B(X, X)
$$

(we denote by the same symbol the dual of the Killing-Cartan form $B(.,$.$) ),$ we find that (9) is nothing but the action of the standard two dimensional Yang-Mills theory. Moreover, if $\varepsilon_{1}$ vanishes, we obtain the $B F$ theory. In both cases, the theory possesses the standard gauge symmetry with respect to the group $G$ and in our picture it is just the residual gauge symmetry (5) that respects the gauge condition $l=(1, X)$. It is given by

$$
X \rightarrow \operatorname{Coad}_{k} X, \quad \eta^{10} \rightarrow k \eta^{10} k^{-1}-\partial_{-} k k^{-1}, \quad \eta^{01} \rightarrow k \eta^{01} k^{-1}-\partial_{+} k k^{-1} .
$$

An important example is given by another double of the same group $G$. We simply take $D(G)=G \times G$ and the invariant form $\langle.,$.$\rangle on its Lie algebra$ $\mathcal{D} \equiv \mathcal{G} \oplus \mathcal{G}$ is given by

$$
\left\langle\left(\alpha_{1}, \alpha_{2}\right),\left(\beta_{1}, \beta_{2}\right)\right\rangle \equiv B\left(\alpha_{1}, \beta_{1}\right)-B\left(\alpha_{2}, \beta_{2}\right),
$$

where $B(.,$.$) is the standard Killing-Cartan form on \mathcal{G}$. Clearly, the diagonal embedding of $G$ into $G \times G$ is isotropic, i.e.

$$
\langle(\alpha, \alpha),(\beta, \beta)\rangle=0 \text {. }
$$


Now we fix the gauge $l=(g, 1)$ and evaluate the action of our Poisson-Lie Yang-Mills theory (2). We obtain

$$
\begin{gathered}
S=I_{B}(g)+ \\
\frac{1}{2 \pi} \int_{\Sigma}\left[B\left(\partial_{+} g g^{-1}, \eta^{10}\right)-B\left(\eta^{01}, g^{-1} \partial_{-} g\right)-B\left(g \eta^{01} g^{-1}, \eta^{10}\right)+B\left(\eta^{01}, \eta^{10}\right)\right]
\end{gathered}
$$

where

$$
I_{B}(g)=\frac{1}{4 \pi} \int B\left(\partial_{+} g g^{-1}, \partial_{-} g g^{-1}\right)+\frac{1}{24 \pi} \int d^{-1} B\left(d g g^{-1},\left[d g g^{-1}, d g g^{-1}\right]\right)
$$

and we have set all coupling constants $\varepsilon_{i}$ to zero. Needless to say, the model thus obtained is the gauged $G / G$ WZNW model with respect to the form $B(.,$.$) . The residual gauge symmetry (5)$ which preserves the gauge $l=(g, 1)$ is now

$$
g \rightarrow k g k^{-1}, \quad \eta^{10} \rightarrow k \eta^{10} k^{-1}-\partial_{-} k k^{-1}, \quad \eta^{01} \rightarrow k \eta^{01} k^{-1}-\partial_{+} k k^{-1} .
$$

Of course, this is the standard gauge symmetry of the $G / G$ WZNW model.

\section{Lu-Weinstein-Soibelman doubles}

The previous two choices of the doubles of $G$ have lead to the well-known gauge theories in two dimensions. We shall now consider another important choice of $D(G)$, where the double of the simple, compact, connected and simply connected group $G$ is the so called Lu-Weinstein-Soibelman Drinfeld double $D_{L W}(G)$ [11]. We shall see that this choice will lead to the theories known as the Poisson- $\sigma$-models [3, 4, 5].

Recall, that a general Drinfeld double [12 is a $2 n$-dimensional real Lie group $D$ whose Lie algebra $\mathcal{D}$ is equipped with an symmetric invariant non-degenerate R-bilinear form $\langle.,$.$\rangle . Moreover, an existence of two n$ dimensional subgroups $G$ and $\tilde{G}$ is required, such that their Lie algebras $\mathcal{G}$ and $\tilde{\mathcal{G}}$ are isotropic with respect to $\langle.,$.$\rangle and \mathcal{D}$ is the direct sum of the vector spaces $\mathcal{G}$ and $\tilde{\mathcal{G}}$.

Clearly, any Drinfeld double $D$ containing $G$ as one of its isotropic subgroups is the double $D(G)$ in the sense described in the introduction. The

converse need not be true, for we may have a double of $G$ which is not the 
Drinfeld double. Inspite of this fact, we refer to the points in our moduli space as to the Poisson-Lie Yang-Mills theories. The reason is the following: if the double is indeed the Drinfeld double then Poisson-Lie brackets are simultaneously induced respectively on the group manifolds $G$ and $\tilde{G}$. In particular, to every such Poisson-Lie structure we associate the corresponding deformation of the ordinary Yang-Mills theory.

The LWS double is simply the complexification (viewed as the real group) $G^{\mathbf{C}}$ of $G$. So, for example, the LWS double of $S U(2)$ is $S L(2, \mathbf{C})$. The invariant non-degenerate form $\langle., .\rangle_{\hbar}$ on the Lie algebra $\mathcal{D}_{l w s}$ of $D_{L W S}$ is given by

$$
\langle x, y\rangle_{\hbar}=\frac{1}{\hbar} \operatorname{Im} B(x, y),
$$

or, in other words, it is just the imaginary part of the Killing-Cartan form divided by a real parameter $\hbar$. Since $G$ is the real form of $G^{\mathbf{C}}$, clearly the imaginary part of $B(x, y)$ vanishes if $x, y \in \mathcal{G}$. Hence, $G$ is indeed isotropically embedded in $G^{\mathbf{C}}$. Note the presence of the parameter $\hbar$ which indicates that we have actually in mind a one-parameter family of doubles.

It turns out that $G^{\mathbf{C}}$ is in fact the Drinfeld double, because $G^{\mathbf{C}}$ is at the same time the double of its another $n$-dimensional subgroup $\tilde{G}$ which coincides with the so called $A N$ group in the Iwasawa decomposition of $G^{\mathbf{C}}$ :

$$
G^{\mathbf{C}}=G A N \text {. }
$$

For the groups $S L(n, \mathbf{C})$ the group $A N$ can be identified with upper triangular matrices of determinant 1 and with positive real numbers on the diagonal. In general, the elements of $A N$ can be uniquely represented by means of the exponential map as follows

$$
\tilde{g}=\mathrm{e}^{\phi} \exp \left[\Sigma_{\alpha>0} v_{\alpha} E_{\alpha}\right] \equiv \mathrm{e}^{\phi} n .
$$

Here $\alpha$ 's denote the roots of $G^{\mathbf{C}}, v_{\alpha}$ are complex numbers and $\phi$ is an Hermitian element] of the Cartan subalgebra of $\mathcal{G}^{\mathbf{C}}$. Loosely said, $A$ is the "noncompact part" of the complex maximal torus of $G^{\mathbf{C}}$. The isotropy of

\footnotetext{
${ }^{1}$ Recall that the Hermitian element of any complex simple Lie algebra $\mathcal{G}^{\mathbf{C}}$ is an eigenvector of the involution which defines the compact real form $\mathcal{G}$; the corresponding eigenvalue is $(-1)$. The anti-Hermitian elements that span the compact real form are eigenvectors of the same involution with the eigenvalue equal to 1 . For elements of $\operatorname{sl}(n, \mathrm{C})$ Lie algebra, the Hermitian element is indeed a Hermitian matrix in the standard sense.
} 
the Lie algebra $\tilde{\mathcal{G}}$ of $\tilde{G}=A N$ follows from (19); the fact that $\mathcal{G}$ and $\tilde{\mathcal{G}}$ generate together the Lie algebra $\mathcal{D}$ of the whole double is evident from (18).

In general, a Poisson bracket $\alpha$ on a manifold $M$ is a smooth section of the bivector bundle on $M$ with vanishing Schouten bracket

$$
[\alpha, \alpha]_{S}=0
$$

Moreover, the Poisson-Lie bracket on a group manifold $G$ has to be compatible with the group multiplication, i.e.

$$
\left\{\triangle F_{1}, \triangle F_{2}\right\}_{G \times G}=\triangle\left\{F_{1}, F_{2}\right\}_{G} .
$$

Here $\triangle F\left(g_{1}, g_{2}\right)=F\left(g_{1} g_{2}\right)$ is the standard coproduct on the algebra of functions on the group manifold and $\{., .\}_{G \times G}$ is the product Poisson structure on $G \times G$ :

$$
\begin{gathered}
\left\{F_{1}(x) G_{1}(y), F_{2}(x) G_{2}(y)\right\}_{G \times G=} \\
=\left\{F_{1}(x), F_{2}(x)\right\}_{G} G_{1}(y) G_{2}(y)+F_{1}(x) F_{2}(x)\left\{G_{1}(y), G_{2}(y)\right\}_{G},
\end{gathered}
$$

where $x$ and $y$ are coordinates on the first and second copy of $G$ respectively. Of course, we have by definition

$$
\left\{F_{1}, F_{2}\right\}_{G} \equiv \alpha\left(d F_{1}, d F_{2}\right) .
$$

Since the bivector bundle on the group manifold is trivializable by the left invariant vector fields, we loose no information about the Poisson-Lie structure $\alpha$ if we trade it for another object, namely a map $\Pi: G \rightarrow \wedge^{2} \mathcal{G}$ defined as follows

$$
\Pi(g) \equiv \Pi_{i j}(g) T^{i} \otimes T^{j} \equiv L_{g^{-1}{ }_{*}} \alpha_{g},
$$

where $T^{i}$ is some basis of $\mathcal{G}, \alpha_{g}$ is the value of the Poisson bivector $\alpha$ at the point $g$ of the group manifold and $L_{g^{-1}}$ is the push-forward map with respect to the left translation by the element $g^{-1}$. The conditions (20) and (21) for the Poisson-Lie structure $\alpha$ translate under (24) to the following conditions for $\Pi(g)$ :

$$
\begin{gathered}
\Pi_{i j}(g)=-\Pi_{j i}(g), \\
\Pi_{k l}\left(\nabla^{k} \Pi_{i j}+\frac{1}{2} f_{i}^{k m} \Pi_{m j}-\frac{1}{2} f_{j}^{k m} \Pi_{m i}\right)+\operatorname{cycl}(l, i, j)=0
\end{gathered}
$$


and

$$
\Pi(g h)=\Pi(h)+\operatorname{Ad}_{h^{-1}} \Pi(g) .
$$

Here $f_{j}^{k m}$ are the structure constants of $\mathcal{G}$ defined as

$$
\left[T^{k}, T^{m}\right]=f_{j}^{k m} T^{j}
$$

and $\nabla^{k}$ is a differential operator acting on functions on $G$ as follows

$$
\left.\nabla^{k} F(g) \equiv \frac{d}{d t} F\left(g \mathrm{e}^{t T^{k}}\right)\right|_{t=0} .
$$

Note that the condition (27) simply says that $\Pi(g)$ is a 1-cocycle in the group cohomology of $G$ with values in $\wedge^{2} \mathcal{G}$.

Now let us introduce an $\hbar$-dependent family of the Poisson-Lie brackets on the group manifolds $G$ and $\tilde{G}=A N$, which are called the LWS Poisson-Lie structures. They are completely determined by the adjoint representation of $G^{\mathbf{C}}$. To describe them, it is convenient to introduce a basis $T^{i}$ in $\mathcal{G}$ and its dual basis $\tilde{T}_{i}$ in $\tilde{\mathcal{G}}$. The duality means the following relation

$$
\left\langle\tilde{T}_{i}, T^{j}\right\rangle_{\hbar}=\delta_{i}^{j}
$$

moreover we have

$$
\left[\tilde{T}_{i}, \tilde{T}_{j}\right]=\tilde{f}_{i j}{ }^{k} \tilde{T}_{k}
$$

A convenient choice of $T^{i}$ 's and of $\tilde{T}_{i}$ 's is given, respectively, by the set $\left(E_{\alpha}+E_{-\alpha}\right), i\left(E_{\alpha}-E_{-\alpha}\right), i H_{i}$ and its dual $-i \hbar E_{\alpha}, \hbar E_{\alpha}, \hbar H_{i}$. Here $H_{i}$ is an (Hermitian) orthonormal basis of the Cartan subalgebra $\mathcal{T}$ with respect to the Killing-Cartan form and $E_{\alpha}, E_{-\alpha}$ are eigenvectors of $\mathcal{T}$ corresponding to roots $\alpha$. Of course, any other basis performs equally well. In fact, we could choose also a basis independent description. It seems to us, however, that in these particular cirmumstances the work with some chosen basis will positively influence the clarity of the exposition. Now for each $\hbar$, define the following matrices (cf. [13])

$$
\tilde{A}_{i}{ }^{j}(\tilde{g})=\left\langle\tilde{g}^{-1} \tilde{T}_{i} \tilde{g}, T^{j}\right\rangle_{\hbar}, \quad \tilde{B}^{i j}(\tilde{g})=\left\langle\tilde{g}^{-1} T^{i} \tilde{g}, T^{j}\right\rangle_{\hbar}, \quad \tilde{g} \in \tilde{G}
$$

and

$$
A^{i}{ }_{j}(g)=\left\langle g^{-1} T^{i} g, \tilde{T}_{j}\right\rangle_{\hbar}, \quad B_{i j}(g)=\left\langle g^{-1} \tilde{T}_{i} g, \tilde{T}_{j}\right\rangle_{\hbar}, \quad g \in G .
$$


It is then a simple matter to check, that the objects

$$
\tilde{\Pi}(\tilde{g})=\tilde{\Pi}^{i j}(\tilde{g}) \tilde{T}_{i} \otimes \tilde{T}_{j}=\tilde{B}^{k i}(\tilde{g}) \tilde{A}_{k}^{j}(\tilde{g}) \tilde{T}_{i} \otimes \tilde{T}_{j}
$$

and

$$
\Pi(g)=\Pi_{i j}(g) T^{i} \otimes T^{j}=B_{k i}(g) A^{k}{ }_{j}(g) T^{i} \otimes T^{j}
$$

define respectively the Poisson-Lie structures on the groups $\tilde{G}$ and $G$ [14]. This means, that the conditions (25),(26) and (27) and their dual analogues are verified. The bivectors (34) and (35) are called the LWS Poisson-Lie structures.

The existence of the global decomposition (18) enables us to define a natural left action of the group $G$ on its dual $\tilde{G}=A N$ which is called the dressing action ([12, 14, 15, 11]). An element $g \in G$ acts on $\tilde{g} \in \tilde{G}$ as follows

$$
{ }^{g} \tilde{g}=\tilde{P}\left(\tilde{g} g^{-1}\right),
$$

where $\tilde{P}$ is the map from $G^{\mathbf{C}}$ onto $\tilde{G}$ induced by the Iwasawa decomposition (18). It is easy to verify that, indeed, (36) defines an action of $G$ i.e.

$$
{ }^{\left(g_{1} g_{2}\right)} \tilde{g}={ }^{g_{1}}\left({ }^{g_{2}} \tilde{g}\right) .
$$

There is a useful formula which clarifies the relation between the dressing transformation (36) and the Poisson-Lie structure $\tilde{\Pi}$ on $\tilde{G}$. Indeed, the infinitesimal action of an element $\beta=\beta_{i} T^{i} \in \mathcal{G}$ on a function $F(\tilde{g})$ is given by

$$
\delta_{\beta} F(\tilde{g})=\tilde{\Pi}^{i j}(\tilde{g}) \beta_{j} \tilde{\nabla}_{i} F(\tilde{g}) \equiv\left(\beta^{\tilde{\sharp} \tilde{g}}\right)^{i} \tilde{\nabla}_{i} F(\tilde{g}) .
$$

The fact that this is really an action, i.e.

$$
\left[\delta_{\beta}, \delta_{\gamma}\right] F(\tilde{g})=-\delta_{[\beta, \gamma]} F(\tilde{g})
$$

follows from the (dual of the) Jacobi identity (26) and from (the infinitesimal version of) the cocycle condition (27):

$$
\tilde{\nabla}_{k} \tilde{\Pi}^{i j}(g)=f_{k}^{i j}-\tilde{f}_{k l}{ }^{i} \tilde{\Pi}^{l j}(\tilde{g})+\tilde{f}_{k l}{ }^{j} \tilde{\Pi}^{l i}(\tilde{g}) .
$$

Before proceeding further, let us study a limit $\hbar \rightarrow 0$. We fix a basis $T^{i}$ in $\mathcal{G}$. Then it is clear that in the limit $\hbar \rightarrow 0$ the commutators of the dual generators $\tilde{T}_{i} \in \tilde{\mathcal{G}}$ tend to zero and $\tilde{G}$ becomes an Abelian group isomorphic 
to $\mathcal{G}^{*}$. In the same sense, the Lie algebra $\mathcal{G}^{\mathrm{C}}$ becomes isomorphic to the semidirect sum of $\mathcal{G}$ and $\tilde{\mathcal{G}}$, where $\mathcal{G}$ acts on $\tilde{\mathcal{G}}$ in the coadjoint way. The dressing action (36) becomes the standard coadjoint action of $G$ on $\mathcal{G}^{*}$ in this limit, the Poisson-Lie structure $\Pi(g)$ vanishes (clearly, $\Pi(g)$ is proportional to $\hbar)$. Moreover, the Poisson-Lie structure $\tilde{\Pi}(\tilde{g})$ becomes nothing but the standard linear Kirillov Poisson bracket on $\mathcal{G}^{*}$. We conclude, that the limit $\hbar \rightarrow 0$ corresponds to the previously considered case of the cotangent bundle $T^{*} G$ as the double $D(G)$. Hence our Poisson-Lie Yang-Mills theory (2) on the LWS double $G^{\mathbf{C}}$ is a 1-parameter deformation of the standard Yang-Mills theory (9). Its action reads

$$
\begin{gathered}
S\left(l, \eta^{10}, \eta^{01}\right)= \\
I(l)+\frac{1}{2 \pi} \int_{\Sigma}\left[\left\langle\partial_{+} l l^{-1}, \eta^{10}\right\rangle_{\hbar}-\left\langle\eta^{01}, l^{-1} \partial_{-} l\right\rangle_{\hbar}-\left\langle l \eta^{01} l^{-1}, \eta^{10}\right\rangle_{\hbar}\right]+\frac{\varepsilon}{2 \hbar^{2}} \int_{\Sigma} \omega \operatorname{tr}\left(l^{\dagger} l-1\right) .
\end{gathered}
$$

Note that we have set all but one $\varepsilon_{i}$ in (2) to zero, and we have chosen canonically the biinvariant term $O_{i}(l)$ where $\operatorname{tr}$ is the trace in the adjoint representation. With an isotropic gauge choice

$$
l=\tilde{P}(l)=\tilde{g} \in \tilde{G},
$$

the action of the Poisson-Lie Yang-Mills theory (41) for $G^{\mathbf{C}}$ becomes

$$
\begin{gathered}
S\left(\tilde{g}, \eta^{10}, \eta^{01}\right)= \\
\frac{1}{2 \pi} \int\left[\left\langle\partial_{+} \tilde{g} \tilde{g}^{-1}, \eta^{10}\right\rangle_{\hbar}-\left\langle\eta^{01}, \tilde{g}^{-1} \partial_{-} \tilde{g}\right\rangle_{\hbar}-\left\langle\eta^{01}, \tilde{g}^{-1} \eta^{10} \tilde{g}\right\rangle_{\hbar}\right]+\frac{\varepsilon}{2 \hbar^{2}} \int \omega \operatorname{tr}\left(\tilde{g}^{\dagger} \tilde{g}-1\right) .
\end{gathered}
$$

Moreover, if we set $\varepsilon=0$ and define

$$
A_{i}^{10} T^{i} \equiv \tilde{A}_{i}{ }^{j}\left(\tilde{g}^{-1}\right) \eta_{j}^{10} T^{i}, \quad A_{i}^{01} T^{i} \equiv \eta_{i}^{01} T^{i} .
$$

then we have (43) as

$$
S\left(\tilde{g}, A^{10}, A^{01}\right)=\frac{1}{2 \pi} \int\left[\left\langle\tilde{g}^{-1} \partial_{+} \tilde{g}, A^{10}\right\rangle_{\hbar}-\left\langle A^{01}, \tilde{g}^{-1} \partial_{-} \tilde{g}\right\rangle_{\hbar}-\tilde{\Pi}(\tilde{g})\left(A^{01}, A^{10}\right)\right],
$$

where $\tilde{\Pi}(\tilde{g})$ is the LWS Poisson-Lie structure (34). Introduce a differential form $A$,

$$
A \equiv A^{10}+A^{01},
$$


then we can rewrite (45) as

$$
S(\tilde{g}, A)=-\frac{1}{2 \pi} \int\left[\left\langle A \wedge \tilde{g}^{-1} d \tilde{g}\right\rangle_{\hbar}+\frac{1}{2} \tilde{\Pi}(\tilde{g})(A \wedge A)\right] .
$$

This is precisely the Poisson $\sigma$-model (1) (written in the left-invariant frame) for the Poisson-Lie group manifold $\tilde{G}$. Of course, we could introduce some coordinates $X^{i}$ and write (47) directly in the form (1). We conclude that for the vanishing coupling constant $\varepsilon=0$, the Poisson-Lie Yang-Mills theory gives the Poisson $\sigma$-model.

The gauge fixing (42) is only partial, the residual group of gauge symmetry (5) consists of the dressing gauge transformations by elements $k\left(\xi_{+}, \xi_{-}\right) \in$ $G$ :

$$
\begin{gathered}
\tilde{g} \rightarrow{ }^{k} \tilde{g}, \\
A^{01} \rightarrow k A^{01} k^{-1}-\partial_{+} k k^{-1}, \\
A^{10} \rightarrow k\left[A^{10}-\left(\left(A^{10}\right)^{\tilde{\sharp} \tilde{g}}+\tilde{g}^{-1} \partial_{-} \tilde{g}\right)^{\sharp k}\right] k^{-1}-\partial_{-} k k^{-1} .
\end{gathered}
$$

Of course, $\sharp_{k}$ is defined in the dual way to (38), namely for $W=W^{i} \tilde{T}_{i} \in \tilde{\mathcal{G}}$ we have

$$
W^{\sharp k}=\left(W^{\sharp k}\right)_{i} T^{i} \equiv \Pi_{i j}(k) W^{j} T^{i} .
$$

In the limit $\hbar \rightarrow 0$ the Poisson-Lie structure $\Pi(k)$ vanishes and (50) becomes the standard gauge transformation law like (11).

The reader may convince himself, that the prescription (48)-(50) defines indeed the action of the gauge group on the triple of the fields $\left(\tilde{g}, A^{10}, A^{01}\right)$. She or he may also directly check the gauge invariance of the action (43) or (47) with respect to the gauge transformation (48)-(50).

Infinitesimal version of the transformations (48)-(50) is given by (38) and by

$$
\begin{gathered}
\delta_{\beta} A_{i}^{01}=-\partial_{+} \beta_{i}-f^{j k}{ }_{i} A_{j}^{01} \beta_{k} ; \\
\delta_{\beta} A_{i}^{10}=-\partial_{-} \beta_{i}-f^{j k}{ }_{i} A_{j}^{10} \beta_{k}-\tilde{f}_{i k}{ }^{l}\left[\left(\tilde{g}^{-1} \partial_{-} \tilde{g}\right)^{k}+\tilde{\Pi}(\tilde{g})^{k m} A_{m}^{10}\right] \beta_{l} .
\end{gathered}
$$

The transformation (38) is the same as in [5] for the case of the Poisson-Lie groups, but (52) and (53) are different. This is actually an interesting issue. Ikeda [3], Schaller\&Strobl [4] and Cattaneo\&Felder [5] have remarked a gauge symmetry of the Poisson $\sigma$-models that closes only on shell if the Poisson structure is not linearly dependent on the coordinates $X^{i}$. The absence of the off shell closure then requires to use the Batalin-Vilkovisky quantization. 
Our gauge symmetry (38), which together with (49) and (50) closes even offshell, acts in the same way on the Poisson manifold as the one in [5]. It is due to this fact that we find plausible to conjecture that the Kontsevich formula can be derived by the standard Faddeev-Popov procedure in the special case of the Poisson-Lie structures.

As an example, consider the double $S L(2, \mathbf{C})$ of $S U(2)$. We choose the basis of $s u(2)$ as

$$
T^{j}=\frac{i}{2} \sigma^{j},
$$

where $\sigma^{j}$ are the Pauli matrices, and the dual basis of $\tilde{\mathcal{G}}$ as

$$
\tilde{T}_{1}=\frac{1}{2} \hbar\left(\begin{array}{ll}
0 & 1 \\
0 & 0
\end{array}\right), \quad \tilde{T}_{2}=\frac{1}{2} \hbar\left(\begin{array}{cc}
0 & -i \\
0 & 0
\end{array}\right), \quad \tilde{T}_{3}=\frac{1}{4} \hbar\left(\begin{array}{cc}
1 & 0 \\
0 & -1
\end{array}\right) .
$$

The coordinate parametrization of the group $\tilde{G}$ is as follows

$$
\tilde{g}=\left(\begin{array}{cc}
\mathrm{e}^{\frac{1}{4} \hbar X 3} & 0 \\
0 & \mathrm{e}^{-\frac{1}{4} \hbar X^{3}}
\end{array}\right)\left(\begin{array}{cc}
1 & \frac{1}{2} \hbar\left(X^{1}-i X^{2}\right) \\
0 & 1
\end{array}\right) .
$$

With these data, the Poisson-Lie Yang-Mills theory (43) becomes

$$
\begin{gathered}
S=\frac{1}{2 \pi} \int \Sigma_{i=1}^{3} C_{i} \wedge d X^{i} \\
+\frac{1}{2 \pi} \int\left\{X^{1} C_{2} \wedge C_{3}+X^{2} C_{3} \wedge C_{1}+\left[\frac{1}{\hbar}\left(1-\mathrm{e}^{-\hbar X^{3}}\right)+\frac{1}{4} \hbar \bar{X} X\right] C_{1} \wedge C_{2}\right\} \\
+\frac{\varepsilon}{2} \int d^{2} \xi\left[\frac{8}{\hbar^{2}}\left(\cosh \frac{1}{2} \hbar X^{3}-1\right)+\bar{X} X \mathrm{e}^{\frac{1}{2} \hbar X^{3}}\right]
\end{gathered}
$$

where we have set

$$
X \equiv X^{1}+i X^{2}, \quad \bar{X} \equiv X^{1}-i X^{2}
$$

The parameter $\varepsilon$ is the coupling constant. Clearly, for $\hbar \rightarrow 0$ we recover the ordinary $S U(2)$ Yang-Mills theory, because the terms in the square brackets in the second and third lines of (57) become respectively $X^{3}$ and $\left(X^{3}\right)^{2}+\bar{X} X$ in this limit. If the coupling constant $\varepsilon$ vanishes, (57) gives the Poisson $\sigma$ model which is the $\hbar$-deformation of the $B F$ theory. 
The infinitesimal dressing transformation on $\tilde{G}$ is generated by the following vector fields

$$
\begin{gathered}
v^{1}=\tilde{\Pi}^{i 1} \tilde{\nabla}_{i}=\left[\frac{1}{\hbar}\left(1-\mathrm{e}^{-\hbar X^{3}}\right)-\frac{1}{4} \hbar \operatorname{Re}(X X)\right] \partial_{X^{2}}-X^{2} \partial_{X^{3}}+\frac{1}{2} \hbar X^{1} X^{2} \partial_{X^{1}}, \\
v^{2}=\tilde{\Pi}^{i 2} \tilde{\nabla}_{i}=\left[-\frac{1}{\hbar}\left(1-\mathrm{e}^{-\hbar X^{3}}\right)-\frac{1}{4} \hbar \operatorname{Re}(X X)\right] \partial_{X^{1}}+X^{1} \partial_{X^{3}}-\frac{1}{2} \hbar X^{1} X^{2} \partial_{X^{2}} \\
v^{3}=\tilde{\Pi}^{i 3} \tilde{\nabla}_{i}=X^{2} \partial_{X^{1}}-X^{1} \partial_{X^{2}} .
\end{gathered}
$$

One can check that those vector fields leave invariant the term $\left[\frac{8}{\hbar^{2}}\left(\cosh \frac{1}{2} \hbar X^{3}-\right.\right.$ $\left.1)+\bar{X} X \mathrm{e}^{\frac{1}{2} \hbar X^{3}}\right]$. We do not write the gauge transformations (49) and (50) explicitely, because the corresponding formulas are cumbersome and not too illuminating anyway. Their basic ingredients are given, however, by components of the Poisson-Lie bivectors. They read

$$
\begin{gathered}
\tilde{\Pi}\left(X^{i}\right)^{12}=-\frac{1}{\hbar}\left(1-\mathrm{e}^{-\hbar X^{3}}\right)+\frac{1}{4} \hbar \bar{X} X, \quad \tilde{\Pi}\left(X^{i}\right)^{23}=-X^{1}, \quad \tilde{\Pi}\left(X^{i}\right)^{31}=-X^{2} ; \\
\Pi(u, v)_{12}=\hbar v \bar{v}, \quad \Pi(u, v)_{23}=\frac{1}{2} \hbar(u v+\bar{u} \bar{v}), \quad \Pi(u, v)_{31}=\frac{1}{2} i \hbar(\bar{u} \bar{v}-u v) .
\end{gathered}
$$

Here an element $g$ of $S U(2)$ is parametrized by two complex coordinates $u, v$ fulfilling $\bar{u} u+\bar{v} v=1$;

$$
g=\left(\begin{array}{cc}
u & -\bar{v} \\
v & \bar{u}
\end{array}\right)
$$

Much of what we said in this section about the LWS doubles remains true in a more general situation. Actually, if the double of a Lie group $G$ is a Drinfeld double $D$, it follows that it exists the dual group $\tilde{G}$ which is also the isotropic subgroup of $D$. If, moreover, every element $l \in D$ can be unambiguously represented as

$$
l=k \tilde{k}, \quad k \in G, \tilde{k} \in \tilde{G}
$$

and the induced map $D \rightarrow G \times \tilde{G}$ is a diffeomorphism, then the Poisson-Lie structures on the groups $G$ and $\tilde{G}$ are again given by the expressions (34) and (35). Of course, one uses the invariant bilinear form that corresponds to the double in question. The Poisson-Lie Yang-Mills theory, with the gauge group based on $G$, corresponding to the double $D$ and with the vanishing coupling constant, is then again given by the Poisson $\sigma$-model (47) where $\tilde{\Pi}$ is the Poisson-Lie structure on $\tilde{G}$. 


\section{The partition function}

The partition function of the ordinary Euclidean Yang-Mills theory has been computed by many methods [1, 2, 16, 17, 18, 19. Here we shall calculate this quantity for the LWS deformation of the Yang-Mills theory introduced in the previous section. We use an appropriate generalization of the method of Blau and Thompson [2, 8].

\subsection{The Wick rotation}

The definition of an Euclidean version of the Poisson-Lie Yang-Mills theory (2) requires some care. The reason is the chiral gauge symmetry (5). If we naively replace $\partial_{-}$by $\partial_{z}$ and $\partial_{+}$by $\partial_{\bar{z}}$ we cannot view the elements $\partial_{z} g g^{-1}$ and $\partial_{\bar{z}} k k^{-1}$ as elements of $\mathcal{G}$ because they are actually the elements of $\mathcal{G}^{\mathbf{C}}$. This suggests that the fields $\eta^{10}$ and $\eta^{01}$ are also independent elements of $\mathcal{G}^{\mathrm{C}}$. This would change, however, the number of degrees of freedom of our theory. We may try to use the standard prescription in gauge theory, namely, take $\eta^{10}$ and $\eta^{01}$ in $\mathcal{G}^{\mathbf{C}}$ and declare $\eta^{01}$ to be an anti-Hermitian conjugate of $\eta^{10}$. This would balance the correct number of degrees of freedom but the independent chiral gauge transformations (5) of $\eta^{10}$ and $\eta^{01}$ would not respect such a constraint.

The way out of the trouble is a partial gauge fixing. We see in the examples (9) and (14) that we can partially fix the gauge (11) or (16) in such a way that the residual gauge symmetry acts in the same way on $\eta^{10}$ and $\eta^{01}$. This makes possible to take $\eta^{10}$ as the anti-Hermitian conjugate of $\eta^{10}$ and indeed this is the standard way how the ordinary Yang-Mills theory and the gauged $G / G$ model are put on the Riemann surface.

Unfortunately, the gauge fixing (42) in the LWS case still leads to the residual gauge symmetry (49) and (50) which acts differently on $\eta^{10}$ and $\eta^{01}$ (or, rather, on $A^{10}$ and $A^{01}$ ). We can consider, however, another gauge fixing which does make possible to define the Euclidean version of the theory?2. It uses the Cartan decomposition [20] of the group $G^{\mathbf{C}}$ which says that every

\footnotetext{
${ }^{2}$ The reason why we did not consider immediately this new gauge fixing is simple: we wanted to make link to the Poisson $\sigma$-model (1) that underlies the Kontsevich formula and this link was explicit in the gauge (42).
} 
element $l \in G^{\mathbf{C}}$ can be represented as

$$
l=p g
$$

where $g \in G$ and $p \in P$. This decomposition is unique. Here our notation is standard; if we consider the set of the Hermitian elements of $\mathcal{G}^{\mathrm{C}}$ (cf. footnote 1 ), we have $\mathcal{P} \equiv i \mathcal{G}$ and

$$
P=\exp \mathcal{P}
$$

The exponential mapping in (67) is one-to-one.

All this makes possible to choose conveniently the new gauge fixing as

$$
l=\hat{P}(l)=p \in P,
$$

where the map $\hat{P}: G^{\mathbf{C}} \rightarrow P$ is induced by the Cartan decomposition (xy). The residual gauge symmetry (5) in this gauge becomes

$p \rightarrow k p k^{-1}, \quad \eta^{10} \rightarrow k \eta^{10} k^{-1}-\partial_{-} k k^{-1}, \quad \eta^{01} \rightarrow k \eta^{01} k^{-1}-\partial_{+} k k^{-1}, \quad k \in G$.

Now it is straightforward to write the Euclidean version of the Poisson-Lie Yang-Mills theory (41) in the gauge (68):

$$
\begin{gathered}
S_{E}\left(p, \eta^{10}, \eta^{01}, \varepsilon, \hbar\right)=I_{E}(p)+ \\
\frac{i}{2 \pi} \int_{\Sigma_{g}}\left[\left\langle\bar{\partial} p p^{-1}, \eta^{10}\right\rangle_{\hbar}-\left\langle\eta^{01}, p^{-1} \partial p\right\rangle_{\hbar}-\left\langle p \eta^{01} p^{-1}, \eta^{10}\right\rangle_{\hbar}\right]+\frac{\varepsilon}{2 \hbar^{2}} \int_{\Sigma_{g}} \omega \operatorname{tr}\left(p^{2}-1\right),
\end{gathered}
$$

where

$$
I_{E}(p)=\frac{i}{4 \pi} \int_{\Sigma_{g}}\left\langle\bar{\partial} p p^{-1}, \partial p p^{-1}\right\rangle_{\hbar}+\frac{i}{24 \pi} \int_{\Sigma_{g}} d^{-1}\left\langle d p p^{-1},\left[d p p^{-1}, d p p^{-1}\right]\right\rangle_{\hbar} .
$$

The gauge symmetry is given by the following transformations

$$
p \rightarrow k p k^{-1}, \quad \eta^{10} \rightarrow k \eta^{10} k^{-1}-\partial k k^{-1}, \quad \eta^{01} \rightarrow k \eta^{01} k^{-1}-\bar{\partial} k k^{-1} .
$$

Remark that we use in (70) and (71) the language of differential forms though we do not indicate explicitly the wedge products between the forms. The operators $\partial$ and $\bar{\partial}$ are the Dolbeault coboundary operators with respect to the chosen complex structure on the Riemann surface $\Sigma_{g}$ ( $g$ indicates the genus of the surface). The forms $\eta^{10}$ and $\eta^{01}$ are respectively the $(1,0)$ and 
$(0,1)$ forms in the Dolbeault complex and the form $\eta^{10}+\eta^{01}$ is in $\mathcal{G} \otimes T^{*} \Sigma_{g}$ and is interpreted as a connection on the (for the simply connected $G$ necessarily) trivial $G$ bundle over $\Sigma_{g}$. In particular, it means that

$$
\eta^{01}=-\left(\eta^{10}\right)^{\dagger}
$$

In other words, $\eta^{10}$ is the anti-Hermitian conjugate of $\eta^{01}$, where the operation $\dagger$ is the Hermition conjugation on $\mathcal{G}^{\mathbf{C}}$ tensored with the complex conjugation on $T^{* \mathbf{C}} \Sigma_{g}$. Note that only the term proportional to the coupling constant $\varepsilon$ depends on the measure on the Riemann surface, which itself is normalized as

$$
\int_{\Sigma_{g}} \omega=1
$$

The reader should avoid a pitfall in understanding the formula (70). It has to do with the fact that the LWS double $D_{L W S}$ of the compact simple connected and simply connected group $G$ is isomorphic to the complexification $G^{\mathbf{C}}$ of $G$. For the purpose of defining the Euclidean version of the Poisson-Lie Yang-Mills theory, we have declared the 1-forms $\eta^{10}$ and $\eta^{01}$ to be the elements of $\mathcal{G}^{\mathbf{C}}$, hence seemingly to be the elements of the Lie algebra of the double. In fact, it is indeed correct to say that in the Euclidean version $\eta^{10}$ and $\eta^{01}$ are the elements of a complexification of the Lie algebra $\mathcal{G}$, but it is not correct to interpret $\eta^{10}$ and $\eta^{01}$ as the elements of the Lie algebra $\mathcal{D}_{\text {lws }}$ of the double. The solution of this apparent paradox is that two different (though mathematically isomorphic) complexifications of $\mathcal{G}$ play role here.

In order to disentangle the two different complexifications, let us work from the very beginning with the real group $D_{L W S}$ as if we did not know that it can be identified with the complexification of $G$. Then consider the complexified group $D_{L W S}^{\mathbf{C}}$ and its Lie algebra $\mathcal{D}_{l w s}^{\mathbf{C}}$. Upon the complexification of $\mathcal{D}_{\text {lws }}$ to $\mathcal{D}_{\text {lws }}^{\mathrm{C}}$, the subalgebra $\mathcal{G} \subset \mathcal{D}_{\text {lws }}$ gets complexified to $\left(\mathcal{G}^{\mathbf{C}}\right)^{\prime}$. We indicate by ' that this complexification is not the same as the complexification $\mathcal{G}^{\mathbf{C}}=\mathcal{D}_{\text {lws }}$. In fact, the forms $\eta^{10}$ and $\eta^{01}$ are to be understood as the elements of $\left(\mathcal{G}^{\mathbf{C}}\right)^{\prime}$ in full agreement with the Euclidean treatment of the coset models (cf. 21]). Of course, the invariant bilinear form $\langle., .\rangle_{\hbar}$ on $\mathcal{D}_{\text {lws }}$ gets extended onto $\mathcal{D}_{l w s}^{\mathbf{C}}$ by bilinearity (not sesquilinearity!). 


\subsection{The measure of the path integral}

We now wish to quantize the theory (70). It actually resembles (the gauging of) the WZNW model on the symmetric space $P$ as defined in [21]. The difference is, however, that there the Killing-Cartan form $B(.,$.$) was used while$ we are using the invariant bilinear form $\left.\langle., .\rangle_{\hbar}\right]^{3}$. The two models have nevertheless some common features. They live both on the symmetric space $P$ which is a contractible manifold diffeomorphic to the Euclidean space $\mathbf{R}^{\operatorname{dim} G}$. This fact means that the $d^{-1}$ of the WZNW 3-form (based on whatever invariant nondegenerate bilinear form) does exist globally. As the consequence, we do not have to extend the map $\Sigma_{g} \rightarrow P$ to a 3-manifold, whose boundary is $\Sigma_{g}$, if we want to determine the contribution of the WZNW-term. Hence the level of the WZNW model (which is equal to $1 / \hbar$ in our case) does not get quantized and it can be an arbitrary positive real number.

We also note that the gauge symmetry (72) is diagonal and hence it is not anomalous. By the way, also the chiral symmetries (5) of the original Poisson-Lie Yang-Mills theory (2) are not anomalous since the Lie algebra $\mathcal{G}$ is isotropic. We may interpret it by saying that also at the quantum level the theory (70) is the gauge fixed version of the Poisson-Lie Yang-Mills theory (41).

The partition function of the model (70) on the genus $g$ Riemann surface is given by the following path integral.

$$
Z(\varepsilon, \hbar, g)=\frac{1}{\operatorname{Vol}\left(G_{\Sigma_{g}}\right)} \int\left(D p D \eta^{10} D \eta^{01}\right)_{g} \exp -S_{E}\left(p, \eta^{10}, \eta^{01}, \varepsilon, \hbar\right),
$$

where the action $S_{E}$ is explicitly written in $(70)$ and $\operatorname{Vol}\left(G_{\Sigma_{g}}\right)$ is the volume of the gauge group. It is natural to expect that the $G$-invariant measures $\left(D \eta^{10} D \eta^{01}\right)_{g}$ and $(D p)_{g}$ should be based on the bilinear form $\langle., .\rangle_{\hbar}$ that underlies the model (41) or (70). On the other hand, there appears an immediate trouble in using $\langle., .\rangle_{\hbar}$ for defining the measure on the fields $\eta^{10}$ and $\eta^{01}$; indeed, these fields are isotropic with respect to $\langle., .\rangle_{\hbar}$, hence it is not clear how to build up a non-zero norm on the field space.

We can circumvent the trouble by borrowing some inspiration from the Lie group theory. The standard measure on a simple complex group $G^{\mathbf{C}}$ is

\footnotetext{
21].

${ }^{3}$ For example, our WZNW action $I_{E}(a)$ vanishes for $a \in A$ which is not the case in
} 
only indirectly defined by the Killing-Cartan form $B(.,$.$) on \mathcal{G}^{\mathbf{C}}$. Actually, people define another $G$-invariant bilinear form [22] as follows

$$
K(X, Y)=B\left(X^{\dagger}, Y\right), \quad X, Y \in \mathcal{G}^{\mathbf{C}}
$$

where we remind that $\dagger$ means the Hermitian conjugation in $\mathcal{G}^{\mathrm{C}}$. Equipped with the form $K(.,$.$) , the Lie algebra \mathcal{G}^{\mathrm{C}}$ becomes an Euclidean space. By left transport of this Euclidean form from the origin of the group manifold $G^{\mathbf{C}}$ everywhere, the Riemannian metric and, hence, the Riemannian measure on $G^{\mathbf{C}}$ is canonically defined. The reasonf for such a construction is simple. With the choice of the positive definite bilinear form $K(.,$.$) , a standartly$ $K$-normalized measure is defined at the same time also for all Lie subgroups of $G^{\mathbf{C}}$.

In our case, there also exists the $G$-invariant way of turning the bilinear form $\langle., .\rangle_{\hbar}$ into a positive definite bilinear form on $\mathcal{G}^{\mathbf{C}}$. For this and also for further purposes it is convenient to fix canonically a real basis of the Lie algebra $\mathcal{D}_{\text {lws }}$ :

$$
\mathcal{D}_{l w s}=\operatorname{Span}_{\mathbf{R}}\left(R^{\alpha}, J^{\alpha}, K^{j}, r_{\alpha}, j_{\alpha}, k_{j}\right)
$$

where

$$
\begin{gathered}
R^{\alpha}=\frac{1}{\sqrt{2}}\left(E_{\alpha}+E_{-\alpha}\right), \quad J^{\alpha}=\frac{-i}{\sqrt{2}}\left(E_{\alpha}-E_{-\alpha}\right), \quad K^{j}=\frac{i 2 H_{\alpha_{j}}}{B\left(H_{\alpha_{j}}, H_{\alpha_{j}}\right)} ; \\
r_{\alpha}=\frac{-i \hbar}{\sqrt{2}}\left(E_{\alpha}+E_{-\alpha}\right), \quad j_{\alpha}=\frac{-\hbar}{\sqrt{2}}\left(E_{\alpha}-E_{-\alpha}\right), \quad k_{j}=\hbar H_{\lambda_{j}} .
\end{gathered}
$$

Here our conventions and normalizations are the same as in 23. This means, in particular,

$$
\begin{gathered}
B\left(E_{\alpha}, E_{-\alpha}\right)=-1, \quad E_{\alpha}^{\dagger}=-E_{-\alpha}, \quad\left[E_{\alpha}, E_{-\alpha}\right]=-H_{\alpha} ; \\
{\left[H, E_{\alpha}\right]=\alpha(H) E_{\alpha}, \quad \alpha(H)=B\left(H_{\alpha}, H\right),}
\end{gathered}
$$

where $H$ is an arbitrary element of the Cartan subalgebra $\mathcal{T}, \alpha_{j}$ 's are the simple roots and $\lambda_{j}$ 's the fundamental weights. We recognize in $-i K^{j}$ 's the simple coroots to which the fundamental weights $\lambda_{j}$ 's are dual.

Note that the (anti-Hermitian) capital generators $R^{\alpha}, J^{\alpha}, K^{j}$ generate the compact real form $\mathcal{G}$ of $\mathcal{D}_{\text {lws }}$. The small generators $r_{\alpha}, j_{\alpha}, k_{j}$ do not

\footnotetext{
${ }^{4}$ We are indebted to P. Delorme for this explanation.
} 
generate a Lie subalgebra of $\mathcal{D}_{\text {lws }}$ but they span the vector subspace in $\mathcal{D}_{\text {lws }}$ that coincides with $\mathcal{P}$ (cf. the discussion between Eqs.(66) , and (67)). The commutation relations of the elements of the basis (xy) give rise to real structure constants thus they define the real Lie algebra $\mathcal{D}_{\text {lws }}$. In this basis, the invariant bilinear form $\langle., .\rangle_{\hbar}$ is given as follows

$$
\left\langle R^{\alpha}, r_{\beta}\right\rangle_{\hbar}=\delta_{\beta}^{\alpha}, \quad\left\langle J^{\alpha}, j_{\beta}\right\rangle_{\hbar}=\delta_{\beta}^{\alpha}, \quad\left\langle K^{i}, k_{j}\right\rangle_{\hbar}=\delta_{j}^{i},
$$

and all other inner products vanish. Thus we see that small generators are in some sense dual to the capital ones, but this decomposition does not give rise to a Manin triple because $\mathcal{P}$ is not a Lie algebra.

Consider an $\mathbf{R}$-linear flip map $\theta, \theta^{2}=1$, defined by changing the capital character into the small one in the canonical basis $(77)\left(\right.$ e.g. $\left.\theta\left(R^{\alpha}\right)=r_{\alpha}\right)$. Then we define the $G$-invariant positive definite bilinear form $K_{\hbar}(.,$.$) as$ follows

$$
K_{\hbar}(X, Y) \equiv\langle\theta(X), Y\rangle_{\hbar}
$$

Note the similarity between (76) and (83); $\theta$ is the analog of $\dagger$ in (76). A $2 n$ dimensional Euclidean volume form $d \mathcal{D}_{l w s}$ on the Lie algebra $\mathcal{D}_{l w s}$, originating from $K_{\hbar}(.,$.$) , is given by$

$$
\begin{gathered}
d \mathcal{D}_{\text {lws }}=\left(\bigwedge_{\alpha \in \Delta_{+}}\left(d R^{\alpha} \wedge d J^{\alpha}\right)\right) \wedge\left(\bigwedge_{j} d K^{j}\right) \wedge\left(\bigwedge_{\alpha \in \Delta_{+}}\left(d r_{\alpha} \wedge d j_{\alpha}\right)\right) \wedge\left(\bigwedge_{j} d k_{j}\right) \\
\equiv d \mathcal{T}^{\perp} \wedge d \mathcal{T} \wedge d \mathcal{A}^{\perp} \wedge d \mathcal{A} .
\end{gathered}
$$

Here $d R^{\alpha}, d J^{\alpha}, d K^{j}, d r_{\alpha}, d j_{\alpha}, d k_{j}$ is by definition the dual basis (of the dual space of the Lie algebra $\mathcal{D}_{\text {lws }}$ ) with respect to the $K_{\hbar \text {-orthonormal basis }}$ $R^{\alpha}, J^{\alpha}, K^{j}, r_{\alpha}, j_{\alpha}, k_{j}$. We use the symbol $K_{\hbar}$, because the volume form on $\mathcal{D}_{\text {lws }}$ computed from $K_{\hbar}(.,$.$) differs the volume form coming from K(.,$.$) by$ a factor $c(\mathcal{G}) \hbar^{\operatorname{dim} \mathcal{G}}$, where $c(\mathcal{G})$ is an $\hbar$-independent constant.

A measure on the gauge fields $\eta=\eta^{10}+\eta^{01}$ coming from $K_{\hbar}(.,$.$) is defined$ by an inner product on the tangent space at each point of the connection space $\eta$ :

$$
\left(\delta \eta_{1}, \delta \eta_{2}\right)=\frac{1}{4 \pi} \int_{\Sigma_{g}} K_{\hbar}\left(\delta \eta_{1} \wedge \hat{,} \delta \eta_{2}\right)
$$

It is independent on the point $\eta$ in the connection space and is gauge invariant by virtue of the $G$-invariance of the form $K_{\hbar}$. It can be easily checked that a 
measure on $\eta$ defined by $K(.,$.$) differs from our measure (85) by a constant$ independent on $\hbar$. This fact will play an important role in what follows.

By using $K_{\hbar}(.,$.$) , we have landed (up to a normalization) on the same$ volume form as the one standardly used in group theory. It is also clear that our measure on $P$ will differ only by the normalization factor from the standard measure on the symmetric space $P$ [24, 25]. Indeed, let us define this measure in the way useful also for further applications.

First of all we define a volume form $d D_{L W S}$ on the $2 n$-dimensional group manifold $D_{L W S}$. We do that by the left transport of the Lie algebra volume form $d \mathcal{D}_{l w s}$. Thus $\left(d D_{L W S}\right)_{l}$ at a point $l \in D_{L W S}$ is now defined as

$$
\left(d D_{L W S}\right)_{l}=L_{l^{-1}}^{*} d \mathcal{D}_{l w s}
$$

where $L_{l^{-1}}^{*}$ is the pull-back of the form $d \mathcal{D}_{l w s}$ (defined in the unit element of the group) by the left translation diffeomorphism. Note that the invariant volume form $d D_{L W S}$ is thus canonically normalized by the bilinear form $K_{\hbar}$.

The measure $d P$ on the symmetric space $P=D_{L W S} / G$ is the most simply defined in the following way: consider the "projection" map $\hat{P}: D_{L W S} \rightarrow$ $P$ defined in (68). Then the integral of an arbitrary function with compact support $f(p)$ on $P$ is defined by the prescription

$$
\int f(p) d P \equiv \frac{1}{V o l(G)} \int\left(\hat{P}^{*} f\right)(l) d D_{L W S},
$$

where $\hat{P}^{*} f$ is the pull-back of the function $f$ by the "projection" map $\hat{P}$ and $\operatorname{Vol}(G)$ is the volume of the compact group $G$. Of course, the measure on the subgroup $G \subset D_{L W S}$ is also standartly $K_{\hbar}$-normalized hence it makes sense to speak about the volume of $G$. In fact, we can readily write the volume form $d G$ on $G$, it is given by

$$
(d G)_{g}=L_{g^{-1}}^{*}\left[d \mathcal{T}^{\perp} \wedge d \mathcal{T}\right] .
$$

We finish this subsection by noting that the measure $(D p)_{g}$ of the path integral (75) is given by the Riemann surface point-wise product of the measures (87).

\subsection{The generalized Weyl integral formula}

Our strategy for computing (75) will be similar as in [2, 8]. It means that we shall first Abelianize the theory by finding a generalized version of the Weyl 
integral formula and then we shall compute the Abelian partition function in the standard way [2]. The non-Abelian origin of the Abelianized theory will be remembered in the determinants produced by the Abelianization procedure.

It turns out that the generalization of the Weyl integral formula, which would work in our setting, indeed exists. It is given in 24 (p.186) and, in more general setting and including the normalization, in [25]. This formula is based on another form of the Cartan decomposition which says that any element $l$ of $G^{\mathbf{C}}$ can be (non uniquely) written as

$$
l=g a k^{-1}, \quad g, k \in G, \quad a \in A .
$$

In particular, it follows from the Cartan decomposition (66) that the elements of $P$ can be represented as

$$
p=k a k^{-1}, \quad k \in G, \quad a \in A .
$$

The ambiguity of this representation of $p$ is clearly parametrized by the elements of the normalizer of $A$ in $G$; we denote this group as $N_{G}(A)$. Evidently, there is a normal subgroup $Z_{G}(A) \subset N_{G}(A)$ containing the elements of $G$ which commute with $A$. This subgroup is called the centralizer of $A$ in $G$ and in our case it coincides with the maximal torus $T$ of $G$. From the fact that $\exp \mathcal{T}=T$ and $\exp i \mathcal{T}=A$, we conclude that the quotient group $N_{G}(A) / Z_{G}(A)$ is nothing but the Weyl group of $\mathcal{G}^{\mathbf{C}}$. Thus the decomposition (90) is unique if we view $k$ as a class in $G / T$ and $a$ as an element of $A_{+}$. Here $A_{+}=\exp \mathcal{A}_{+}$and $\mathcal{A}_{+}$is the fundamental domain (=the Weyl chambre) of the action of the Weyl group on $\mathcal{A}=i \mathcal{T}$. For this unique parametrization of $P$, we can infer the generalized Weyl integral formula 24, 25] which holds for the functions satisfying

$$
f(p)=f\left(k p k^{-1}\right), \quad k \in G .
$$

It reads

$$
\int_{P} f(p) d P=\frac{\operatorname{Vol}(G)}{\operatorname{Vol}(T)} \int_{A^{+}} J(a) f(a) d A,
$$

where

$$
J(a)=\Pi_{\alpha \in \Delta} \frac{1}{2 \hbar}\left|a^{\alpha}-a^{-\alpha}\right| .
$$


With a parametrization

$$
a=\exp \phi^{j} k_{j}=\exp \hbar \phi^{j} H_{\lambda_{j}} \equiv \exp \hbar \phi, \quad \phi \in \mathcal{A}_{+}
$$

we have

$$
J(\phi)=\Pi_{\alpha \in \Delta_{+}} \frac{\sinh ^{2}(\hbar \alpha(\phi))}{\hbar^{2}} .
$$

The set of all roots of $\mathcal{G}^{\mathrm{C}}$ is denoted as $\Delta$, the set of positive roots as $\Delta_{+}$. The measure $d A$ is the standard measure on $A \subset D_{L W S}$ in the sense discussed above; it is given by the $K_{\hbar}$-normalized volume form

$$
(d A)_{a}=L_{a^{-1}}^{*} d \mathcal{A} .
$$

The volume $\operatorname{Vol}(G)$ is computed with respect to the standard measure $d G$, defined in (88), and $\operatorname{Vol}(T)$ with respect to

$$
(d T)_{t}=L_{t^{-1}}^{*} d \mathcal{T}, \quad t \in T .
$$

The formula (95) gives the Jacobian $J(\phi)$; for $\hbar=1$ it coincides with the Jacobian in [24, 25]. Note that the limit $\hbar \rightarrow 0$ makes sense and it produces the Jacobian which arises in the Weyl integral formula for the Lie algebra $\mathcal{G}$ [2]. In what follows, we shall use a notation often used in the world of quantum groups; i.e.

$$
[x]_{\hbar}=\frac{\sinh \hbar x}{\hbar}
$$

for an arbitrary number $x$. With this notation, the Jacobian $J(\phi)$ becomes the product of the $q$-numbers:

$$
J(\phi)=\prod_{\alpha \in \Delta_{+}}[\alpha(\phi)]_{\hbar}^{2}
$$

Let us now give the proof of (95). It is clear that the integral $\int f(p) d P$ in the l.h.s. of (92) reduces to some integral over $A_{+}$since both the function $f(p)$ and the measure $d P$ are invariant with respect to the conjugation by elements of $G$ (the latter fact follows from the simultaneous left and right $G$-invariance of $\left.d D_{L W S}\right)$. It is not difficult to find the volume form corresponding to this integration. For this, define first a map $\hat{A}_{+}: P \rightarrow A_{+}$that associates to every $p \in P$ the element $a \in A_{+}$under the Cartan decomposition (90). Clearly, 
the function $f(p)$ on $P$ satisfying (91) is the pull-back of some function $\tilde{f}(a)$ on $A_{+}$by the map $\hat{A}_{+}$. We are looking for a function $J(a)$ such that

$$
\int \tilde{f}(a) J(a) d A_{+}=\frac{1}{\operatorname{Vol}(G) \operatorname{Vol}(G / T)} \int\left(\hat{P}^{*} \hat{A}_{+}^{*} \tilde{f}\right)(l) d D_{L W S}
$$

Here $\operatorname{Vol}(G / T)$ is calculated from the measure on the homogeneous space $G / T$ defined in a similar way as the measure on $D_{L W S} / G$ (cf.(87)). It then follows

$$
\operatorname{Vol}(G / T)=\frac{\operatorname{Vol}(G)}{\operatorname{Vol}(T)}
$$

We see from (100) that

$$
J(a)\left(d A_{+}\right)_{a}=i_{\left\{{ }^{L} \mathcal{T}_{a}^{\perp}\right\}} i_{\left\{{ }^{R} \mathcal{G}_{a}\right\}}\left(d D_{L W S}\right)_{a},
$$

where the multivector $\left\{{ }^{R} \mathcal{G}_{a}\right\}$ is defined as

$$
\left\{{ }^{R} \mathcal{G}_{a}\right\} \equiv \bigwedge_{\alpha \in \Delta_{+}}\left({ }^{R} R_{a}^{\alpha} \wedge{ }^{R} J_{a}^{\alpha}\right) \wedge\left(\bigwedge_{j}{ }^{R} K_{a}^{j}\right)
$$

and the multivector $\left\{{ }^{L} \mathcal{T}_{a}^{\perp}\right\}$ as

$$
\left\{{ }^{L} \mathcal{T}_{a}^{\perp}\right\} \equiv \bigwedge_{\alpha \in \Delta_{+}}\left({ }^{L} R_{a}^{\alpha} \wedge{ }^{L} J_{a}^{\alpha}\right)
$$

Here e.g. ${ }^{R} J_{a}^{\alpha}$ realizes the right action of the generator $J^{\alpha}$ of $\mathcal{G}$ on the group manifold $D_{L W S}$ at the point $a$ and the multivector $\left\{{ }^{L} \mathcal{T}_{a}^{\perp}\right\}$ corresponds to the left action of $\mathcal{T}^{\perp}$ on $D_{L W S}$ at the same point $a$. Clearly, $i_{V} \omega$ denotes the insertion of the multivector $V$ into the form $\omega$. Every such a generator, say $R J_{a}^{\alpha}$, can be written as

$$
{ }^{R} J_{a}^{\alpha}=L_{a *} J^{\alpha}, \quad J^{\alpha} \in \mathcal{G},
$$

where $L_{a *}$ is the push-forward map corresponding to the left transport. In a similar way, we have

$$
\left\{{ }^{L} \mathcal{T}_{a}^{\perp}\right\}=R_{a *}\left\{\mathcal{T}^{\perp}\right\}=L_{a *}\left(A d_{a^{-1}}\left\{\mathcal{T}^{\perp}\right\}\right)
$$

Thus we immediately arrive at

$$
J(a)\left(d A_{+}\right)_{a}=i_{R_{a^{*}}\left\{\mathcal{T}^{\perp}\right\}} i_{L_{a^{*}}\{\mathcal{G}\}} L_{a^{-1}}^{*}\left(d \mathcal{T}^{\perp} \wedge d \mathcal{T} \wedge d \mathcal{A}^{\perp} \wedge d \mathcal{A}\right)=
$$




$$
=L_{a^{-1}}^{*}\left(i_{A d_{a^{-1}}\left\{\mathcal{T}^{\perp}\right\}}\left(d \mathcal{A}^{\perp} \wedge d \mathcal{A}\right)\right) .
$$

We calculate

$$
\begin{aligned}
& A d_{a^{-1}} R^{\alpha}=R^{\alpha} \cosh \hbar \alpha(\phi)+j_{\alpha} \frac{\sinh \hbar \alpha(\phi)}{\hbar} ; \\
& A d_{a^{-1}} J^{\alpha}=J^{\alpha} \cosh \hbar \alpha(\phi)-r_{\alpha} \frac{\sinh \hbar \alpha(\phi)}{\hbar} .
\end{aligned}
$$

Inserting (108) and (109) into (107) and taking into account (84) and (107), it follows

$$
J(\phi)=\Pi_{\alpha \in \Delta_{+}} \frac{\sinh ^{2}(\hbar \alpha(\phi))}{\hbar^{2}}=\Pi_{\alpha \in \Delta_{+}}[\alpha(\phi)]_{\hbar}^{2} .
$$

\subsection{The maximal torus bundles}

Consider the gauge

$$
p=a=\exp \hbar \phi, \quad \phi=\phi^{j} H_{\lambda_{j}} \quad \phi \in \mathcal{A}_{+}
$$

and introduce a pair of real ghosts $c_{\alpha}^{r}, c_{\alpha}^{j}$ for each positive root $\alpha$. By taking into account (70), (92), (110) and (111), the formula (75) for the partition functions becomes

$$
\begin{aligned}
& Z(\varepsilon, \hbar, g)=\frac{1}{\operatorname{Vol}\left(T_{\Sigma_{g}}\right)} \int\left(D \phi D \eta^{10} D \eta^{01} D c^{r} D c^{j}\right)_{g} \exp -\varepsilon \int \omega \sum_{\alpha \in \Delta}[\alpha(\phi)]_{\hbar}^{2} \\
& \exp -\frac{i}{2 \pi} \int\left\{d \phi^{j} \wedge A_{j}+\sum_{\alpha \in \Delta_{+}}\left([\alpha(\phi)]_{\hbar} B_{\alpha} \wedge C_{\alpha}+\omega[\alpha(\phi)]_{\hbar}^{2} c_{\alpha}^{r} c_{\alpha}^{j}\right)\right\}
\end{aligned}
$$

Here

$$
\begin{aligned}
& \eta^{10}=A^{10}{ }_{m} K^{m}+B^{10}{ }_{\alpha} R^{\alpha}+C^{10}{ }_{\alpha} J^{\alpha} ; \\
& \eta^{01}=A^{01}{ }_{m} K^{m}+B^{01}{ }_{\alpha} R^{\alpha}+C^{01}{ }_{\alpha} J^{\alpha} ;
\end{aligned}
$$

and the component forms $A_{m}^{10}, B_{\alpha}^{10}, C_{\alpha}^{10}$ and $A_{m}^{01}, B_{\alpha}^{01}, C_{\alpha}^{10}$ are pairwise complex conjugated forms in $T^{* \mathrm{C}} \Sigma_{g}$.

Note that the term $I_{E}(p=a)$ in (70) gives no contribution to (112). This seems to be a trivial fact because $\mathcal{A}$ is commutative and isotropic with respect to $\langle.,\rangle_{\hbar}$. However, the isotropy and commutativity together with the cohomological triviality of the WZNW term explains the vanishing of $I_{E}(a)$. In the case of the $G / G$ gauged WZNW model the WZNW term is 
not cohomologically trivial and it may and does contribute [2, 27. This can be easily understood by realizing that in the cohomologically nontrivial situation one has to consider the mappings extended to three-dimensional domain of which $\Sigma_{g}$ is the boundary. This extension need not respect the (isotropic and/or commutative) gauge choice on $\Sigma_{g}$.

Our formula (112) almost coincides with the result of the Abelianization of the ordinary Yang-Mills theory (formula (2.58) of [2]). Up to a trivial overall $2 \pi$ normalization? the only difference in the action consists in replacing the "ordinary" numbers $\alpha(\phi)$ of Blau and Thompson by our quantum numbers $[\alpha(\phi)]_{\hbar}$. Moreover, the measure of our path integral differs from that of the ordinary Yang-Mills case only by an $\hbar$-independent constant. This constant originates from the difference between our measure-defining form $K_{\hbar}(.,$.$) and the ordinary Yang-Mills measure-defining form K(.,$.$) , where$ the $\hbar$-dependent part of this difference is already taken into account in the Jacobian $J(\phi)$ or, in other words, in the ghost part of the action. Moreover, also the $2 \pi$ renormalization of the measure of the gauge fields play role (cf. our Eq.(85) and Eq.(2.11) of [2]). We shall eventually use a freedom to renormalize our measure-defining bilinear form by all these $\hbar$-independent constants in such a way that the limit $\hbar \rightarrow 0$ in (112) gives a correctly normalized ordinary Yang-Mills partition function.

There is a very important aspect of exploiting the (generalized) Weyl integral formula in the ordinary Yang-Mills case [2] and also in our PoissonLie Yang-Mills setting. It has to do with the following fact: the validity of the Cartan decomposition (90) of an arbitrary element $p \in P$ does not imply that the smooth mapping $p(\bar{z}, z): \Sigma_{G} \rightarrow P$ can be smoothly decomposed as

$$
p(\bar{z}, z)=g(\bar{z}, z) a(\bar{z}, z) g(\bar{z}, z)^{-1}, \quad g(\bar{z}, z) \in G, \quad a(\bar{z}, z) \in A_{+} .
$$

Of course, the mappings $g(\bar{z}, z)$ and $a(\bar{z}, z)$ do exist but it is by no means guaranteed that they be smooth. This fact has serious implications for the proper meaning of the formula (112). Strictly speaking, we cannot choose the gauge (111) smoothly. Then what do we mean by Eq. (112)?

A hard work of Blau and Thompson [8] was needed for solving this problem in the case of the ordinary Yang-Mills theory (and also in the case of the $G / G$ gauged WZNW model). Fortunately, we can fully rely on their

\footnotetext{
${ }^{5}$ Blau and Thompson have chosen the overall normalization to be in accord with the fixed point theorems while we are using the standard WZNW normalization.
} 
results also in the LWS case because $P$ is diffeomorphic to the Lie algebra $\mathcal{G}$ of $G$ and this (exponential) diffeomorphism commute with conjugations by the elements of $G$. In fact, we have

$$
P=\exp i \mathcal{G}
$$

This means that we map $p(\bar{z}, z) \in P$ into $\mathcal{G}$ by taking the inverse of the mapping (116) and then we apply the Blau-Thompson diagonalization i.e.

$$
-i \ln p(\bar{z}, z)=g(\bar{z}, z) t(\bar{z}, z) g^{-1}(\bar{z}, z), \quad t(\bar{z}, z) \in \mathcal{C}_{+} \subset \mathcal{T} .
$$

Here $\mathcal{C}_{+}=-i \mathcal{A}_{+}$is the Weyl chamber in $\mathcal{T}$. By multiplying (117) by $i$ and then exponentiating, we arrive at the seeken Cartan decomposition

$$
p(\bar{z}, z)=g(\bar{z}, z) a(\bar{z}, z) g^{-1}(\bar{z}, z), \quad a(\bar{z}, z)=e^{i t(\bar{z}, z)} \in A_{+} .
$$

The analysis [8] of the diagonalization of the type (117) can be translated into our context along the lines above and it gives the following results: If $\ln p(\bar{z}, z)$ is a smooth map from the Riemann surface $\Sigma_{g}$ into a subset of regular elements of $\mathcal{G}$ then

1) The smooth decomposition (115) can always be achieved locally on $\Sigma_{g}$.

2) The diagonalized map $a(\bar{z}, z)$ can always be chosen to be smooth globally. 3) Non-trivial $T$-bundles on $\Sigma_{g}$ are the obstructions to finding smooth functions $g(\bar{z}, z)$ globally. In particular, if there are no nontrivial principal $G$ bundles on $\Sigma_{g}$ (like in our case), all isomorphism classes of torus bundles appear as obstructions.

4) The gauge field path integral should include a sum over the $\mathcal{T}$-connections on all isomorphism classes of $T$-bundles on $\Sigma_{g}$.

Actually, the point 4) shows in which sense we should understand the formula (112).

It is not so difficult to understand intuitively, what is going on here. If the function $g(\bar{z}, z)$ is not smooth somewhere, then passing from $p(\bar{z}, z)$ to $a(\bar{z}, z)$ is a singular gauge transformation and the $g(\bar{z}, z)$-transformed connection field $\eta$ becomes singular. It is well-known that singular connections can be sometimes interpreted as connections on nontrivial bundles (see [2, 26] for examples). 
The condition that $-i \ln p(\bar{z}, z)$ is a regular element of the Lie algebra $\mathcal{G}$ may seem inconspicuous but it is in fact crucial for the proper definition of the path integral. By restricting our space of fields $p(\bar{z}, z)$ to those verifying the condition of the regularity, we make a certain choice. We can certainly understand it simply as a part of a plausible definition of the path integral, because, as it was shown in [8] such regular maps with values in the Lie algebra $\mathcal{G}$ are generic. In order to corroborate this choice we give two arguments (a more detailed discussion is provided in [2, 8] and it is directly relevant also to our Poisson-Lie Yang-Mills case):

First of all, the restriction to the regular maps gives the correct answer for the ordinary Yang-Mills case, which is confirmed by alternative methods of calculation 11, 16, 17, 18, 19. Secondly, the non-regular $\phi$ 's are anyway automatically suppressed from the path integral since the Jacobian $J(\phi)$, originating from the generalized Weyl integral formula, vanishes for them. Indeed, the Jacobian $J(\phi)$ vanishes if and only if $-i \phi$ is not a regular element of $\mathcal{T}$. This can be seen directly from the definition of the regularity; an element $X$ of the Cartan subalgebra $\mathcal{T}$ is regular iff it satisfies a condition $\operatorname{det}_{\mathcal{T} \perp}(\operatorname{ad}(X)) \neq 0$ where the notation means that the $\operatorname{ad}(X)$ operator is restricted to $\mathcal{T}^{\perp}$. Of course, if this $\operatorname{ad}(X)$ determinant vanishes then there exists a root $\alpha$ such that $\alpha(X)=0$. The latter fact implies the vanishing of the Jacobian $J(X)$. The opposite direction can be also easily proved.

Following closely [2], we shall now perform the path integral over the affine space of Abelian connections $A_{j}$. The maximal torus bundles are parametrized by the monopole numbers $\left(n_{1}, \ldots, n_{\text {rankG }}\right)$ given by

$$
\int_{\Sigma_{g}} F_{j}(A)=2 \pi n_{j}
$$

where $A=A_{j} K^{j}$ is a connection on the bundle and $F(A)=F_{j}(A) K^{j}$ is its curvature. For each set of the monopole numbers we choose one connection $A^{c l}$ which we call the classical monopole solution. It fulfils

$$
d A_{c l}=2 \pi n_{j} K^{j} \omega
$$

Now every connection $A$ can be written as

$$
A=A_{c l}+A_{q}
$$


where the "quantum part" $A_{q}$ of the connection is a 1 -form on $\Sigma_{g}$ with values in $\mathcal{T}$. The path integral over $A$ then becomes the sum over the monopole numbers and the path integral over $A_{q}$. The latter imposes a constraint

$$
d \phi=0 .
$$

The details of this procedure which, of course, must be accompanied by an appropriate gauge fixing and ghost integration are presented in [2] Eqs. (2.71)-(2.80). Their calculation applies to our case without any change. Thus the integral over $\phi$ reduces to a finite integral over the constant mode of $\phi$ and we shall note the corresponding measure $d \phi$ instead of $D \phi$. Of course, the integral over $d \phi$ is taken over the (interior of the) Weyl chamber $\mathcal{A}_{+}=-i \mathcal{C}_{+}$. The result is

$$
\begin{gathered}
Z(\varepsilon, \hbar, g)=\int\left(d \phi D B D C D c^{r} D c^{j}\right) \exp -\varepsilon \sum_{\alpha \in \Delta}[\alpha(\phi)]_{\hbar}^{2} \\
\sum_{n_{1}, \ldots, n_{\text {rank }}} \exp \left\{i n_{j} \phi^{j}-\frac{i}{2 \pi} \int_{\Sigma_{g}} \sum_{\alpha \in \Delta_{+}}\left([\alpha(\phi)]_{\hbar} B_{\alpha} \wedge C_{\alpha}+\omega[\alpha(\phi)]_{\hbar}^{2} c_{\alpha}^{r} c_{\alpha}^{j}\right)\right\} .
\end{gathered}
$$

Here we have also used Eq. (74).

The path integral over the $B, C$ fields and over the ghosts was performed in [2] in generality, which covers not only the ordinary Yang-Mills and the $G / G$ gauged WZNW case but also our Poisson-Lie Yang-Mills case. The functions $M_{\alpha}$ defined in Eq. (B.5) of [2] are in our context $M_{\alpha}=[\alpha(\phi)]_{\hbar}$. We infer from (B.23) of [2] that

$$
Z(\varepsilon, \hbar, g)=\int d \phi\left(\Pi_{\alpha \in \Delta_{+}}[\alpha(\phi)]_{\hbar}\right)^{2-2 g} \sum_{n_{1}, \ldots, n_{r a n k \mathcal{G}}} \exp \left\{i n_{j} \phi^{j}-\varepsilon \sum_{\alpha \in \Delta}[\alpha(\phi)]_{\hbar}^{2}\right\} .
$$

Contrary to the $G / G$ case and in accord with the ordinary Yang-Mills theory, there is no shift of a "level" $1 / \hbar$. The last step of calculation consists in performing the $d \phi$ integral. We use the well-known formula

$$
\frac{1}{2 \pi} \sum_{n_{j}} e^{i n_{j} \phi^{j}}=\sum_{m^{j}} \delta\left(\phi^{j}-2 \pi m^{j}\right) .
$$

Here on the right-hand-side we recognize the periodic $\delta$ function. The usual $2 \pi$ factor in this formula is understood to be hidden in the definition of the 
measure $d \phi$. Substituting the expression (125) into (124), we arrive at

$$
\begin{gathered}
Z(\varepsilon, \hbar, g)= \\
\frac{1}{|W|} \sum_{m^{1}, \ldots, m^{\text {rank }}}\left(\Pi_{\alpha \in \Delta_{+}}\left[2 \pi m^{j} \alpha\left(H_{\lambda_{j}}\right)\right]_{\hbar}\right)^{2-2 g} \exp -\varepsilon \sum_{\alpha \in \Delta}\left[2 \pi m^{j} \alpha\left(H_{\lambda_{j}}\right)\right]_{\hbar}^{2} .
\end{gathered}
$$

Note that here we have conveniently extended the domain of definition of $\phi$ from $\mathcal{A}_{+}$to whole $\mathcal{A}$ and we compensated this by factoring the volume $|W|$ of the Weyl group. We now interpret the summation over $m^{j}$ as summation over the weight lattice of $\mathcal{G}$. The latter is defined as

$$
\Lambda=\mathbf{Z}\left[\lambda_{1}, \ldots, \lambda_{r}\right]
$$

where $r=\operatorname{rank\mathcal {G}}$ and $\lambda_{i}$ are the fundamental weights. We set $\lambda=m^{j} \lambda_{j}$ and we rewrite (126) as

$Z(\varepsilon, \hbar, g)=\frac{1}{|W|} \sum_{\lambda+\rho \in \Lambda_{r}} \Pi_{\alpha \in \Delta_{+}}\left([B(\alpha, \lambda+\rho)]_{2 \pi \hbar}\right)^{2-2 g} \exp -\varepsilon \sum_{\alpha \in \Delta}[B(\alpha, \lambda+\rho)]_{2 \pi \hbar}^{2}$

Note the shift by the Weyl vector $\rho=\frac{1}{2} \sum_{\Delta_{+}} \alpha=\sum_{j} \lambda_{j}$. Since we anyway sum up over the whole weight lattice, this shift can be interpreted as a pure change of the summation variable. Another important remark concerns the notation $\Lambda_{r}$ in (128). By this we mean that we sum only over the regular points of the weight lattice in accord with the discussion above. There is a simple criterion to decide whether an element of the weight lattice is regular or not. In fact, the non-regular elements are precisely those which are located on the walls of the Weyl chambers.

The formula (128) is our final result for the partition function of the LWS Poisson-Lie Yang-Mills theory, or for $\varepsilon=0$, of the Poisson $\sigma$-model corresponding to the LWS Poisson-Lie structure on $\tilde{G}=A N$. We see that, indeed, we can interpret this partition function as the series over the $q$ numbers. In the limit $\hbar \rightarrow 0$, our result agrees with the ordinary Yang-Mills partition function Eq. (2.86) of [2]. To see this, we just have to use the well-known identity 23]

$$
B(x, y)=\sum_{\alpha \in \Delta} B(x, \alpha) B(\alpha, y), \quad x, y \in \mathcal{G}^{*}
$$

for $x=y=\lambda+\rho$. 


\subsection{The Verlinde formula}

Let us rewrite our formula (128) for the partition function $Z(\varepsilon, \hbar, g)$ for the case $\varepsilon=0$ :

$$
Z(\varepsilon=0, q, g)=\frac{1}{|W|} A^{1-g} \sum_{\lambda+\rho \in \Lambda_{r}} \Pi_{\alpha \in \Delta}\left|1-q^{(\alpha, \lambda+\rho)}\right|^{1-g} .
$$

Here $q=\exp 2 \pi \hbar B(\psi, \psi), A$ is a normalization constant to be discussed later and the bilinear form $(.,$.$) on the dual of \mathcal{T}$ is defined by the following rescaling of the Killing-Cartan form:

$$
(X, Y)=\frac{2}{B(\psi, \psi)} B(X, Y) .
$$

Here $\psi$ denotes the longest root.

In our construction, $q$ was a real parameter, nevertheless, we shall now consider $Z(0, q, g)$ as a function of a complex $q$. Strictly speaking, if $q$ is complex, we cannot say apriori whether $Z(0, q, g)$ is a partition function of some theory. We shall see, however, that for $q$ being a root of unity, $Z(0, q, g)$ can be (almost) interpreted as the partition function of the $G / G$ gauged WZNW model. As it is well-known, the latter is given by the Verlinde formula [7] which is a finite sum and not a series. In which sense can we say that our series (130) gives the Verlinde formula for $q$ being a root of unity?

The point is that for $q$ equal to a root of unity, say $q^{k}=1$, the expression under the summation symbol in (130) acquires the affine Weyl symmetry. The affine Weyl group [28] is a semidirect product of the standard Weyl group and of the coroot lattice. The action of the Weyl group on the weight lattice is standard and since the product $\Pi_{\alpha}$ in (130) is taken over all roots, the expression (130) is Weyl invariant (this follows from an idempotency and Killing-Cartan orthogonality of Weyl reflections). An element $\beta^{\vee}$ of the coroot lattice acts on the weight lattice as

$$
\lambda_{\beta^{\vee}}=\lambda+k \frac{1}{2} B(\psi, \psi) B\left(\beta^{\vee}, .\right)=\lambda+k\left(\beta^{\vee}, .\right)^{*} .
$$

Here use the symbol $(., .)^{*}$ for the form on $\mathcal{T}$ dual to $(.,$.$) .$

Recall that for a root $\beta$, the $\operatorname{coroot} \beta^{\vee} \in \mathcal{T}$ is defined by

$$
\beta^{\vee}=\frac{2 H_{\beta}}{B(\beta, \beta)} .
$$


The affine Weyl symmetry of (130) for $q^{k}=1$ is now obvious because

$$
q^{\left(\alpha, \lambda_{\beta} \vee\right)}=q^{(\alpha, \lambda)+k \alpha\left(\beta^{\vee}\right)}=q^{(\alpha, \lambda)} .
$$

The last equality follows from the fact that $\alpha\left(\beta^{\vee}\right)$ is integer, as the result of the contraction of $\alpha$ (which is also an element of the weight lattice) with the coroot $\beta^{\vee}$. Actually, for $q^{k}=1$, the expression (130) makes sense only if the weights lying on affine Weyl orbits of the non-regular weights are excluded from $\Lambda_{r}$. It is precisely in this sense that we understand (130).

We conclude that, for $q^{k}=1$, the series (130) can be written as a summation over the fundamental domain of the affine Weyl group multiplied by its (infinite) volume. It is because of this infinite volume renormalization that we have said above that $Z(0, q, g)$ can be almost interpreted as the partition function of the $G / G$ gauged WZNW model. The fundamental domain of the action of the affine Weyl group is often referred to as the (Weyl) alcove and it contains those elements $\lambda$ of the standard Weyl chamber that fulfil

$$
\lambda\left(\psi^{\vee}\right)<k
$$

Here $\psi^{\vee}$ is the coroot of the longest root. For $G=S U(r+1)$, the condition (135) translates into

$$
\sum_{j=1}^{r} m^{j}<k, \quad m_{j}>0
$$

where $\lambda=\sum_{j} m^{j} \lambda_{j}$ are the dominant weights sweeping the Weyl chamber.

Eq. (130) with the summation restricted to the alcove is nothing but the Verlinde formula. Its correct normalization can be achieved by adding to the action (70) a suitable "counterterm" of the form const $\int_{\Sigma_{g}} R$, where $R$ is the curvature on the Riemann surface.

As we have already remarked, it is not clear from our derivation whether $Z(0, q, g)$ is a partition function of some theory if $q$ is not real. On the other hand, the result for $q$ equal to a root of unity suggests that it is indeed so because the partition function of the $G / G$ gauged WZNW model is given by the Verlinde formula. It might be that this fact is related to an observation made by Alekseev, Schaller and Strobl [29]. They remarked that, modulo some $\delta$-function relict of the WZNW term, the $G / G$ gauged WZNW model can be represented as the Poisson $\sigma$-model where the Poisson structure on $G$ is a complex bivector. If $q$ and, hence, $\hbar$ has a non-vanishing imaginary part 
then in our context the real Poisson bivector $\tilde{\Pi}(\tilde{g})$ gets rescaled by a complex Planck constant $\hbar$ and it becomes complex too. However, there is a crucial difference between our theory and that of Alekseev et al. Our LWS Poisson $\sigma$-model (or the Poisson-Lie Yang-Mills theory with the vanishing coupling

constant) lives on the dual group $\tilde{G}=A N$ while the Poisson $\sigma$-model of 29 lives on the compact group $G$. Inspite of this, the partition functions for particular q's happen to coincide! This suggests that a sort of a quantum Poisson-Lie T-duality [13, 30] between the two topological $\sigma$-models takes place here.

\section{Outlook}

An open field for future investigations is the study of the correlation functions of the Poisson-Lie Yang-Mills theories. It seems to be very plausible that, like in the ordinary Yang-Mills case, one can identify observables whose correlators are insertion independent. It would be also interesting to calculate exactly the three-point boundary correlator of the LWS Poisson $\sigma$-model on the disc. It is known [5] that the perturbation expansion in $\hbar$ gives the Kontsevich formula. If this correlator can be computed by a closed formula, we might attempt to consider a convergence in $\hbar$. Thus $\hbar$ would make sense not only as the formal expansion parameter.

A more algebraic problem consists in attempting a classification of all doubles $D(G)$ of a connected simple compact group $G$. This would lead to a generalization of the classification [31] of the Poisson-Lie structures on $G$.

The Verlinde formula was recently associated to the quantum double of a finite group [32]. Although we have dealt with with the classical double of a Lie group, it would be certainly worth looking at possible relations of the two constructions.

It would be desirable to produce a derivation of the partition function by an alternative method. For example, the gluing and pasting procedure of [16]. It may seem problematic to use this approach because of problems with the definition of the WZNW action on surfaces with boundaries. On the other hand we note that in the LWS Poisson-Lie Yang-Mills case the WZNW term is cohomologically trivial therefore this problem should be accessible. Of course, this last remark is pertinent also for the very definition of the Poisson-Lie Yang-Mills theory on the Riemann surfaces with boundary and 
for the implications concerning the Kontsevich formula.

\section{Acknowledgement}

I am grateful to Patrick Delorme for many interesting discussions and valuable comments.

\section{References}

[1] E. Witten, Commun. Math. Phys. 141 (1991) 153

[2] M. Blau and G. Thompson, Lectures on 2d gauge theories: Topological aspects and path integral techniques, in Proceedings of the 1993 Trieste Summer School on High Energy Physics and Cosmology (eds. E. Gava et al.), World Scientific, Singapore (1994) 175, hep-th/9310144

[3] N. Ikeda, Ann. Phys. 235 (1994) 435

[4] P. Schaller and T. Strobl, Mod. Phys. Lett. A9 (1994) 3129; Poisson$\sigma$-models: A generalization of 2d Gravity-Yang-Mills systems, hepth/9411163, in Proceedings of the International Workshop on 'Finite Dimensional Integrable Systems', Eds. A.N. Sissakian and G.S. Pogosyan, Dubna 1995, 181-190; A brief introduction to Poisson $\sigma$-models, hepth/9507020, in Proceedings Schladming 1995

[5] A.S. Cattaneo and G. Felder, A path integral approach to the Kontsevich quantization formula, math.QA/9902090

[6] M. Kontsevich, Deformation quantization of Poisson manifolds I, $q-\operatorname{alg} / 9709040$

[7] E. Verlinde, Nucl. Phys. B300 (1988) 360

[8] M. Blau and G. Thompson, Commun.Math.Phys. 171 (1995) 639

[9] C. Klimčík and P. Ševera, Nucl. Phys. B488 (1997) 653

[10] A.Polyakov and P.B.Wiegmann, Phys. Lett. B311 (1983) 549 
[11] J.-H. Lu and A. Weinstein, J. Diff. Geom. 31 (1990) 510; Ya.S. Soibelman, Algebra Analiz 2 (1990) 190; V.G. Drinfeld, unpublished

[12] V.G. Drinfeld, Quantum groups, in Proceedings ICM, Berkeley (1986) 708; F. Falceto and K. Gawędzki, J. Geom. Phys. 11 (1993) 251; A. Yu. Alekseev and A.Z. Malkin, Commun. Math. Phys. 162 (1994) 147

[13] C. Klimčík and P. Ševera, Phys. Lett. B351 (1995) 455

[14] H. Flaschka and T. Ratiu, A convexity theorem for Poisson actions of compact Lie groups, preprint IHES/M/95/24 (1995)

[15] M.A. Semenov-Tian-Shanski, Dressing transformations and Poisson-Lie group actions, in Publ.Res. Inst. Math. Sci. Kyoto Univ. 51 (1985), 1237

[16] M. Blau and G. Thompson, Int. J. Mod.Phys. A7 (1992) 3781

[17] E. Witten, Commun.Math.Phys. 141 (1991) 153

[18] B. Rusakov, Mod. Phys. Lett. A5 (1990) 693

[19] A. Hirshfeld and T. Schwarzweller, Path Integral Quantization of the Poisson $\sigma$-model, hep-th/9910178

[20] D. P. Zhelobenko and A.I. Stern, Representations of Lie groups, Moscow, Nauka (1983) p.116, in Russian

[21] K. Gawȩdzki and A. Kupianen, Nucl. Phys. B320 (1989) 625

[22] Harish-Chandra, J. Func. Anal. 19 (1975) 104

[23] J.F. Cornwell, Group theory in physics II, Academic Press (1984), chapter 13

[24] S. Helgason, Groups and Geometric Analysis, Academic Press (1984), p. 186

[25] P. Delorme, Invent. Math. 105 (1991) 305

[26] C. Klimčík, Commun. Math. Phys. 199 (1998) 257 
[27] K. Gawędzki, WZW conformal field theory, in Constructive Quantum Field Theory II, (eds. G. Velo and A. Wightman), Plenum, New York (1990) 89

[28] A. Pressley and G. Segal, Loop groups, Clarendon Press (1986), p.65; K. Gawędzki, Conformal Field Theory, unpublished book

[29] A.Yu. Alekseev, P. Schaller and T. Strobl, Phys.Rev. D52 (1995) 7146

[30] A.Yu. Alekseev, C. Klimčík and A.A. Tseytlin, Nucl. Phys. B458 (1996) 430

[31] Ya.S. Soibelman, Dokl. AN SSSR 307 (1989) 41; Z. Levendorskii and Ya.S. Soibelman, Commun. Math. Phys. 139 (1991) 141

[32] T.H. Koornwinder, B.J. Schroers, J.K. Slingerland and F.A. Bais, Fourier transform and the Verlinde formula for the quantum double of a finite group, math.QA/9904029 